\title{
The Millimeter Array
}

\author{
Robert L. Brown ${ }^{1}$ \\ National Radio Astronomy Observatory, 520 Edgemont Road, \\ Charlottesville, VA 22903-2475
}

\begin{abstract}
The Millimeter Array, under development in the United States, is a fast and flexible telescope capable of imaging the thermal sky at a resolution equal to that specified in the design goals of the Hubble Space Telescope. The MMA is a project of the U.S. National Science Foundation, and it has been highlighted as one of the four major U.S. initiatives in astronomy for the decade of the 1990s. Construction will begin toward the end of the decade at a site soon to be chosen.
\end{abstract}

\section{Overview}

The Millimeter Array (MMA) is a synthesis imaging telescope under development in the United States for more than ten years. The concept for the MMA was developed in a series of meetings in which the scientific needs of millimeter astronomy were distilled to the following:

- sub-arcsecond imaging at $115 \mathrm{GHz}$ and higher frequencies;

- wide-field imaging, mosaicing;

- rapid imaging, "snapshots" of high fidelity;

- sensitive imaging to high frequency (to $\sim 350 \mathrm{GHz}$ );

- simultaneous broadband, multi-band capability;

- comprehensive "single dish" capability.

An array meeting these requirements has to have exceptionally good instantaneous $(u, v)$-coverage-a requirement for a large number of antennas - and it must be flexible and reconfigurable. For mosaicing or wide-field imaging of molecular clouds, the solar disk, or the disks of nearby galaxies, the array antennas will be packed together in a compact configuration. Each antenna will be used to acquire both interferometer and total power, single-dish data. For high angular resolution observations of protostellar or protoplanetary disks or the nuclei of active galaxies, the antennas will be configurable along the locus of an ellipse approximately $2 \times 3$ kilometers in extent. In either case it will be possible for the MMA to observe in two or more frequency bands simultaneously.

The MMA as proposed to the National Science Foundation in 1990 is described in Table 1. An artists concept of part of the array in one of its extended

\footnotetext{
${ }^{1}$ The National Radio Astronomy Observatory is operated by Associated Universities, Inc., under cooperative agreement with the National Science Foundation.
} 
configurations is shown in Figure 1.

Table 1. The MMA - Summary of Instrumental Parameters

\begin{tabular}{ll}
\hline Array - & \\
Number of Antennas: & 40 \\
Total Collecting Area: & $2010 \mathrm{~m}^{2}$ \\
Angular Resolution: & $0{ }^{\prime \prime} 07 \lambda_{(\mathrm{mm})}$ \\
Antennas - & $8.0 \mathrm{~m}$ \\
Diameter: & $\lambda / 40 \mathrm{at} 1 \mathrm{~mm}$ \\
Precision: & $1 / 20 \mathrm{beamwidth}$ \\
Pointing: & \\
Transportable & \\
Configurations - & $70 \mathrm{~m}$ \\
Compact: & $250 \mathrm{~m}, 900 \mathrm{~m}$ \\
Intermediate (2): & $3 \mathrm{~km}$ \\
High Resolution: & $195-366 \mathrm{GHz}$ \\
Frequencies - & $30-50 \mathrm{GHz}$ \\
Emphasis on: & $68-115 \mathrm{GHz}$ \\
Broadband Coverage: & $130-183 \mathrm{GHz}$ \\
& Simultaneous multi-band operation \\
Versatility: &
\end{tabular}

\section{Design Priorities}

The operational plan for the MMA is the same as that for other NRAO instruments, namely it will be a facility open to all astronomers with scientific programs chosen on the basis of peer review and no other criterion. The nature of such an operation places special demands on the design of the array. In particular it emphasizes once again the need for the array to be fast so that a large number of research programs can be accommodated annually. But it also serves to underscore the need for the array design to favor reliability, ease of maintenance, and equipment replacement, and the necessity to locate the array on a site that provides as much unobstructed observing as possible.

Finally the array design concept, and the array site choice, will be optimized so as to minimize the operational cost of the array over the life of the instrument. This latter criterion has several ramifications, one of the more important being provision in the array design for user-provided or special purpose equipment to be added to the array expeditiously. If done properly, such a provision maximizes the scientific resources and minimizes the cost and complexity of research not only for the MMA but for the community as a whole. 


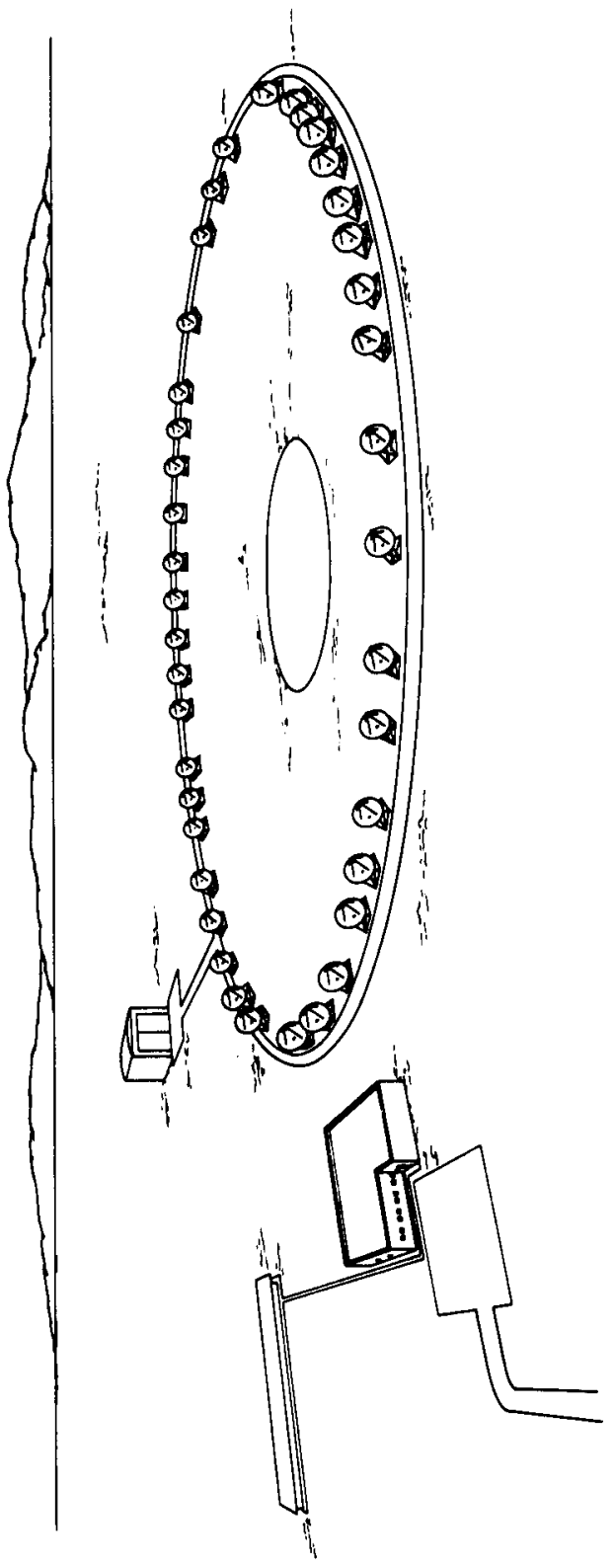

Figure 1. An artist's concept of the Millimeter Array in one of its longer configurations. Antenna pads are shown where the antennas will be clustered close together in the mosaicing configuration. 


\section{MMA Research and Development Phase}

The MMA will begin a more detailed three year design phase in 1994; a plan for this effort has been given to the NSF at their request. The following activities will be carried out in this design phase.

- Site Evaluation and Acquisition. Three possible sites for the MMA are being studied. One in the Magdalena mountains near Socorro, NM, one near Springerville, AZ, and one on Mauna Kea, HI. In the next year the sites will be ordered in priority based on atmospheric transparency and stability measurements and on an evaluation of costs.

- Antenna Design. The MMA antenna is being designed to be exceptionally stiff so that it may be switched rapidly (every ten seconds) between source and calibrator. If such rapid switching is possible the fraction of time the array can be made coherent at the highest frequencies and over the largest baselines exceeds 50 percent of the total time available. We are currently working with a slant-axis design which not only supports rapid switching but is also compact, easy to transport, and easy to pack closely with other antennas in the mosaicing array configurations (see Figure 2).

- SIS Mixer Development. We are designing SIS mixers for the MMA bands at $130 \mathrm{GHz}$ and higher that are simple, free of all mechanical tuners, easy to replicate and maintain. Initial results (Figure 3) for tunerless $1 \mathrm{~mm}$ mixers have resulted in a sensitivity equal to that of our best mixers with tuners and equal also to the needs of the MMA.

- HFET Amplifier Development. For all MMA frequencies below $118 \mathrm{GHz}$ we will use heterostructure field effect transistor amplifiers (HFET). In addition to being broadband and inherently single sideband, the cryogenic cooling of these devices is simple with proven reliability. The projected performance of the millimeter-wave HFET amplifiers is equal to that of the best SIS devices (Figure 4).

- Local Oscillator Design and Prototype;

- IF Processor Design;

- Signal Transmission System Prototype;

- Definition of Monitor and Control Software;

- AIPS++ Computing;

- Correlator, FX, and XF Evaluation;

- Cryogenics Prototype System and Reliability Tests;

- Prototype Receiver and Reliability Tests.

\section{Project Schedule}

Toward the end of the three-year MMA research and development phase we will provide the National Science Foundation with (1) all the results of our work; (2) the ordered list of preferred sites; and (3) the revised array construction and operation cost. Pending approval of the NSF and the U.S. Congress construction could begin in 1997 or 1998 , with completion approximately six years later. 


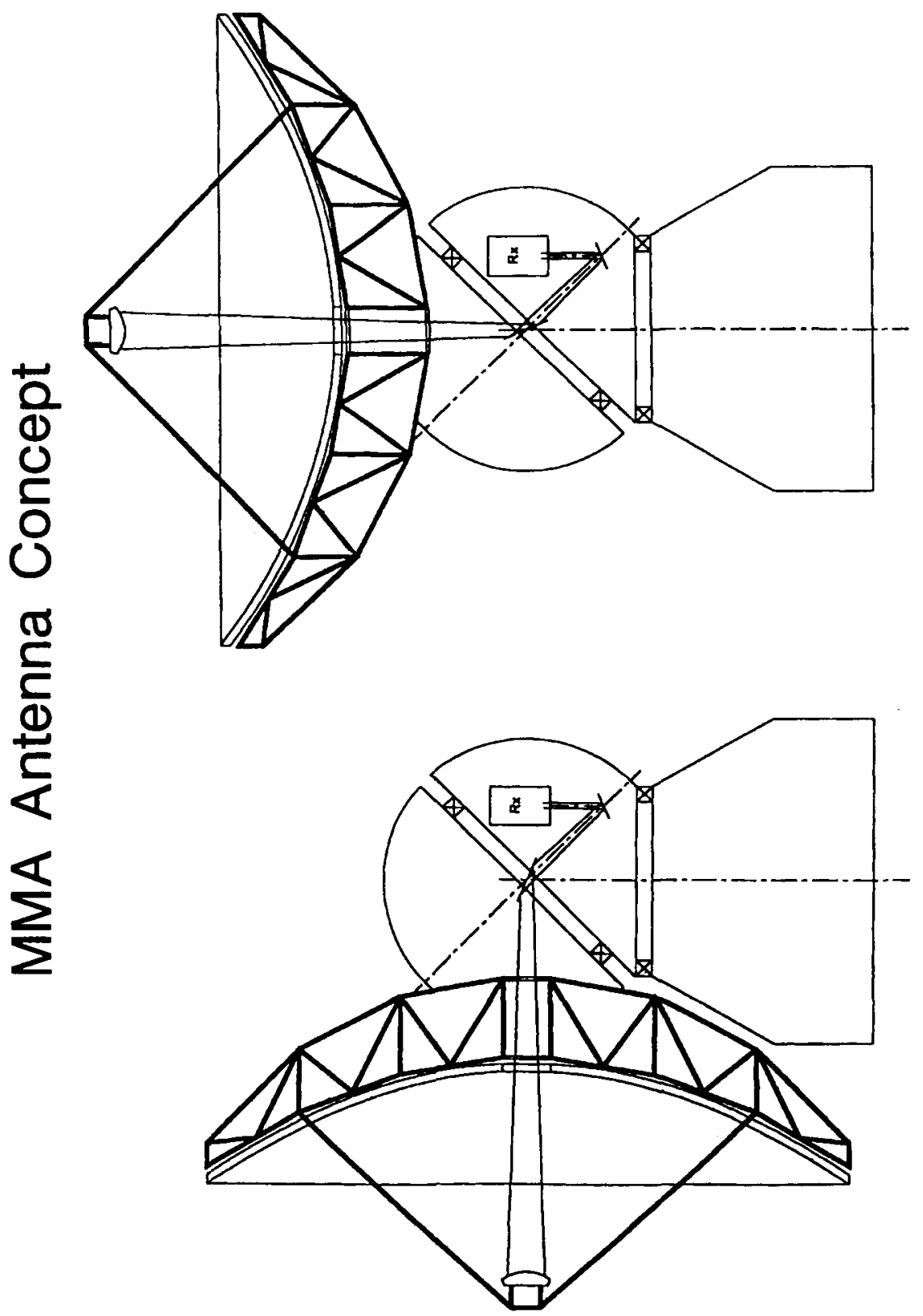

Figure 2. The slant-axis design concept for the MMA antenna. 


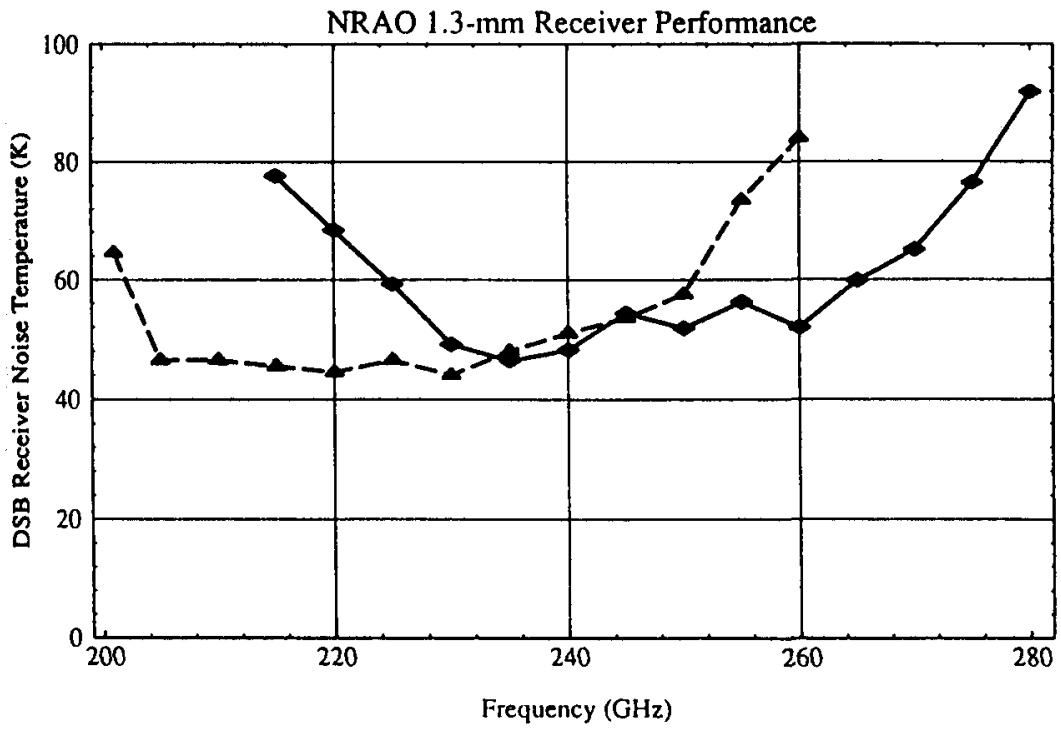

Figure 3. Performance of the NRAO $1 \mathrm{~mm}$ tunerless SIS mixer compared to the performance of the SIS mixer with backshort and bias mechanical tuners.

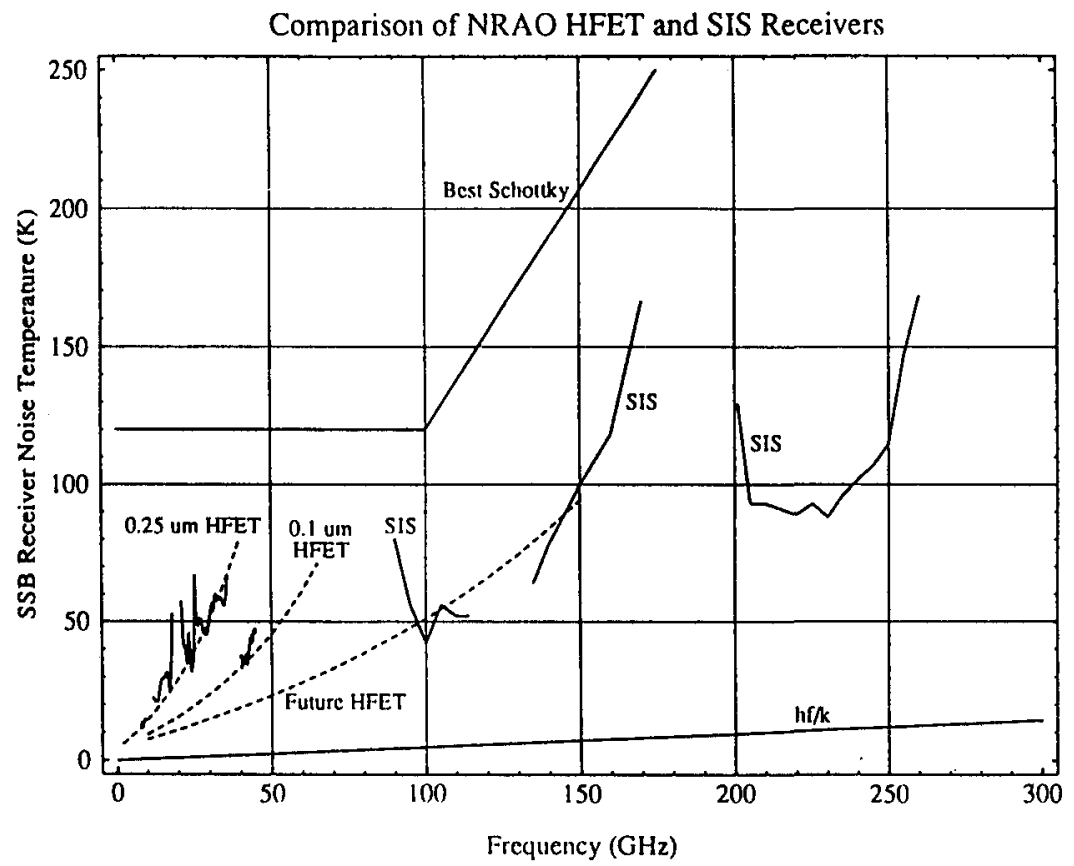

Figure 4. Anticipated performance of a millimeter-wave HFET amplifier. Also shown is the achieved performance of other devices in this frequency range. 


\section{DISCUSSION}

K. Y. Lo

1) Have your OVRO maps been corrected for primary beam?

2) Your results imply $\mathrm{CO}$ emission being within $50 \mathrm{kms}^{-1}$ of each other, but spread over many kpc's, which is most remarkable. Have you considered the possibility of side-lobe response affecting the results?

$\underline{\text { R. Brown }}$ The OVRO maps have not had a primary beam correction applied. We are looking into the possibility of sidelobe contamination by changing the map grid, the taper and so forth. This work is still in progress.

$\underline{\text { R. Ekers }}$ How did you arrive at the decision to use $408 \mathrm{~m}$ dishes?

R. Brown The $408 \mathrm{~m}$ antennas is the result of a cost-minimization analysis. We fixed the desired total collecting area of the array and then optimized (1) the imaging speed of the array, a quantity that is proportional to $(\mathrm{nD})^{2}$ where $\mathrm{n}$ is the number of antennas and $D$ is their diameter and (2) the point source sensitivity, a quantity which is proportional to $\mathrm{nD}^{2}$. The minimum cost solution for these two optimizations is $408 \mathrm{~m}$ antennas. 